\title{
An Introduction to Artificial Neural Networks
}

Daniel Rivero

This presentation is an introduction to the world of Artificial Neural Networks. Nowadays, Artificial Neural Networks are systems that have been very successful in many different problems, so their study is very useful for researchers in many different environments. Specifically, in the biomedical field, knowledge of Artificial Neural Networks is currently of great importance given the large number of applications they have, especially in the intelligent processing of images and signals. In this presentation we will review from the simplest models such as ADALINE to more complex models such as the multilayer perceptron, leaving the way open to start learning Deep Learning techniques. A historical introduction is given and the main algorithms of operation are reviewed, but special emphasis is placed on practical applications, especially in the biomedical field. 